PROCEEDINGS OF THE

AMERICAN MATHEMATICAL SOCIETY

Volume 139, Number 2, February 2011, Pages 461-471

S 0002-9939(2010)10484-7

Article electronically published on September 23, 2010

\title{
UNIVERSAL INEQUALITIES FOR EIGENVALUES OF A CLAMPED PLATE PROBLEM ON A HYPERBOLIC SPACE
}

\author{
QING-MING CHENG AND HONGCANG YANG
}

(Communicated by Matthew J. Gursky)

\begin{abstract}
In this paper, we investigate universal inequalities for eigenvalues of a clamped plate problem on a bounded domain in an $n$-dimensional hyperbolic space. It is well known that, for a bounded domain in the $n$-dimensional Euclidean space, Payne, Pólya and Weinberger (1955), Hook (1990) and Chen and Qian (1990) studied universal inequalities for eigenvalues of the clamped plate problem. Recently, Cheng and Yang (2006) have derived the Yangtype universal inequality for eigenvalues of the clamped plate problem on a bounded domain in the $n$-dimensional Euclidean space, which is sharper than the other ones. For a domain in a unit sphere, Wang and Xia (2007) have also given a universal inequality for eigenvalues. For a bounded domain in the $n$ dimensional hyperbolic space, although many mathematicians want to obtain a universal inequality for eigenvalues of the clamped plate problem, there are no results on universal inequalities for eigenvalues. The main reason that one could not derive a universal inequality is that one cannot find appropriate trial functions. In this paper, by constructing "nice" trial functions, we obtain a universal inequality for eigenvalues of the clamped plate problem on a bounded domain in the hyperbolic space. Furthermore, we can prove that if the first eigenvalue of the clamped plate problem tends to $\frac{(n-1)^{4}}{16}$ when the domain tends to the hyperbolic space, then all of the eigenvalues tend to $\frac{(n-1)^{4}}{16}$.
\end{abstract}

\section{INTRODUCTION}

Let $M$ and $D$ denote an $n$-dimensional complete Riemannian manifold and a bounded domain with boundary $\partial D$ in $M$, respectively. We consider the Dirichlet eigenvalue problem of the biharmonic operator, the so-called clamped plate problem, which describes vibrations of a clamped plate:

$$
\left\{\begin{array}{l}
\Delta^{2} u=\Gamma u, \quad \text { in } D, \\
\left.u\right|_{\partial D}=\left.\frac{\partial u}{\partial \nu}\right|_{\partial D}=0
\end{array}\right.
$$

Received by the editors January 27, 2009.

2010 Mathematics Subject Classification. Primary 35P15, 58G40.

Key words and phrases. Eigenvalue, universal inequality for eigenvalues, hyperbolic space, biharmonic operator and a clamped plate problem.

The first author's research was partially supported by a Grant-in-Aid for Scientific Research from JSPS.

The second author's research was partially supported by the NSF of China and the Fund of the Chinese Academy of Sciences.

(C)2010 American Mathematical Society Reverts to public domain 28 years from publication 
where $\Delta^{2}$ is the biharmonic operator in $M$ and $\frac{\partial}{\partial \nu}$ denotes the outward normal derivative on $\partial D$.

When $M=\mathbf{R}^{n}$, for the clamped plate problem, Payne, Pólya and Weinberger [14] and [15] established a universal inequality for eigenvalues. They obtained

$$
\Gamma_{k+1}-\Gamma_{k} \leq \frac{8(n+2)}{n^{2}} \frac{1}{k} \sum_{i=1}^{k} \Gamma_{i}
$$

Hile and Yeh [10] improved the above result to

$$
\sum_{i=1}^{k} \frac{\Gamma_{i}^{1 / 2}}{\Gamma_{k+1}-\Gamma_{i}} \geq \frac{n^{2} k^{3 / 2}}{8(n+2)}\left(\sum_{i=1}^{k} \Gamma_{i}\right)^{-1 / 2} .
$$

Furthermore, Hook [11] and Chen and Qian [3] proved the following inequality:

$$
\frac{n^{2} k^{2}}{8(n+2)} \leq\left[\sum_{i=1} \frac{\Gamma_{i}^{1 / 2}}{\Gamma_{k+1}-\Gamma_{i}}\right] \sum_{i=1}^{k} \Gamma_{i}^{1 / 2}
$$

Ashbaugh in [1] has pointed out whether one can establish inequalities for eigenvalues of the clamped plate problem which are analogs of the inequalities of Yang for eigenvalues of the Dirichlet eigenvalue problem of the Laplacian. In [6], Cheng and Yang have solved the problem of Ashbaugh affirmatively; that is, they have proved the following:

$$
\Gamma_{k+1}-\frac{1}{k} \sum_{i=1}^{k} \Gamma_{i} \leq\left[\frac{8(n+2)}{n^{2}}\right]^{1 / 2} \frac{1}{k} \sum_{i=1}^{k}\left[\Gamma_{i}\left(\Gamma_{k+1}-\Gamma_{i}\right)\right]^{1 / 2} .
$$

By making use of Chebyshev's inequality, it is not hard to prove that (1.5) implies (1.4).

When $M$ is a unit sphere, Wang and Xia [16] have also given a universal inequality. They have proved

$$
\sum_{i=1}^{k}\left(\Gamma_{k+1}-\Gamma_{i}\right)^{2} \leq \frac{8(n+2)}{n^{2}} \sum_{i=1}^{k}\left(\Gamma_{k+1}-\Gamma_{i}\right)\left(\Gamma_{i}^{1 / 2}+\frac{n^{2}}{2 n+4}\right)\left(\Gamma_{i}^{1 / 2}+\frac{n^{2}}{4}\right) .
$$

When $M$ is a hyperbolic space $H^{n}(-1)$, although many mathematicians want to derive a universal inequality for eigenvalues, there are no results on the universal inequalities for eigenvalues of the clamped plate problem (1.1). For a bounded domain in $H^{n}(-1)$, a main reason that one could not derive a universal inequality for eigenvalues is that one cannot find an appropriate trial function. In this paper, we find "nice" trial functions. By making use of them, we infer a universal inequality for eigenvalues of the eigenvalue problem (1.1).

Theorem 1.1. Let $\Gamma_{i}$ denote the $i^{\text {th }}$ eigenvalue of the clamped plate problem (1.1) on a bounded domain $D$ in $H^{n}(-1)$. Then, we have

$$
\sum_{i, j=1}^{k}\left(\Gamma_{k+1}-\Gamma_{i}\right)^{2} \leq 24 \sum_{i, j=1}^{k}\left(\Gamma_{k+1}-\Gamma_{i}\right)\left\{\Gamma_{i}^{\frac{1}{2}}-\frac{(n-1)^{2}}{4}\right\}\left\{\Gamma_{j}^{\frac{1}{2}}-\frac{(n-1)^{2}}{6}\right\} .
$$

Furthermore, we have the following Yang-type universal inequality for eigenvalues: 
Corollary 1.2. Let $\Gamma_{i}$ denote the $i^{\text {th }}$ eigenvalue of the clamped plate problem (1.1) on a bounded domain $D$ in $H^{n}(-1)$. Then, we have

$$
\sum_{i, j=1}^{k}\left(\Gamma_{k+1}-\Gamma_{i}\right)^{2} \leq 24 \sum_{i, j=1}^{k}\left(\Gamma_{k+1}-\Gamma_{i}\right)\left(\Gamma_{i}-\frac{(n-1)^{4}}{16}\right) .
$$

Remark 1.3. For a buckling problem on a bounded domain in the hyperbolic space, a universal inequality for eigenvalues will be given in a forthcoming paper. Recently, Cheng, Ichikawa and Mametsuka 4 have obtained a universal inequality for eigenvalues of the clamped plate problem on a bounded domain in a complete Riemannian manifold. This occurred after we completed this paper.

For the Dirichlet eigenvalue problem of the Laplacian on a bounded domain in $H^{n}(-1)$, McKean [13] (cf. [2] and [9]) proved that the first eigenvalue $\lambda_{1} \geq \frac{(n-1)^{2}}{4}$ and $\lim _{D \rightarrow H^{n}(-1)} \lambda_{1}=\frac{(n-1)^{2}}{4}$. In [8], Cheng and Yang have proved that all of the eigenvalues of the Laplacian must tend to $\frac{(n-1)^{2}}{4}$ when the domain tends to $H^{n}(-1)$. From the Corollary 1.2 and the recursion formula in Cheng and Yang [7, we have the following:

Theorem 1.4. Let $\Gamma_{i}$ denote the $i^{\text {th }}$ eigenvalue of the clamped plate problem (1.1) on a bounded domain $D$ in $H^{n}(-1)$. If $\lim _{D \rightarrow H^{n}(-1)} \Gamma_{1}=\frac{(n-1)^{4}}{16}$, then, for any $k$, we have

$$
\lim _{D \rightarrow H^{n}(-1)} \Gamma_{k}=\frac{(n-1)^{4}}{16} .
$$

\section{Proofs of the TheOREMS}

In this section, we shall prove our results.

For convenience, we will use the upper half-plane model of the hyperbolic space; that is,

$$
H^{n}(-1)=\left\{\vec{x}=\left(x_{1}, x_{2}, \cdots, x_{n}\right) \in \mathbf{R}^{n} ; x_{n}>0\right\}
$$

with the standard metric

$$
d s^{2}=\frac{\left(d x_{1}\right)^{2}+\left(d x_{2}\right)^{2}+\cdots+\left(d x_{n}\right)^{2}}{x_{n}^{2}} .
$$

In this case, by a simple computation, we have the Laplacian in $H^{n}(-1)$ :

$$
\Delta=x_{n}^{2} \sum_{j=1}^{n} \frac{\partial^{2}}{\partial x_{j} \partial x_{j}}+(2-n) x_{n} \frac{\partial}{\partial x_{n}} .
$$

From the above formula, we have the following lemma:

Lemma 2.1. Defining $f_{i}=x_{i}$, for $i=1,2, \cdots, n-1, f_{n}=\frac{1}{x_{n}}$ and $f=\log x_{n}$, we have

$$
\begin{aligned}
& \Delta f_{i}=0, \text { for } i=1,2, \cdots, n-1, \\
& \Delta f_{n}=n f_{n} \\
& \Delta f=1-n
\end{aligned}
$$


Proof of Theorem 1.1. Let $u_{i}$ be the $i^{\text {th }}$ orthonormal eigenfunction corresponding to the eigenvalue $\Gamma_{i}, i=1,2, \cdots, k$; that is, $u_{i}$ satisfies

$$
\left\{\begin{array}{l}
\Delta^{2} u_{i}=\Gamma_{i} u_{i}, \quad \text { in } D, \\
\left.u_{i}\right|_{\partial D}=\left.\frac{\partial u_{i}}{\partial \nu}\right|_{\partial D}=0, \\
\int_{D} u_{i} u_{j}=\delta_{i j}, \text { for any } i, j .
\end{array}\right.
$$

For the function $f=\log x_{n}$, we have

$$
|\nabla f|^{2}=\nabla f \cdot \nabla f=1, \quad \Delta f=1-n .
$$

We define functions

$$
\varphi_{i}=f u_{i}-\sum_{j=1}^{n} a_{i j} u_{j},
$$

with $a_{i j}=\int_{D} f u_{i} u_{j}$. Then, we have

$$
\begin{gathered}
\left.\varphi_{i}\right|_{\partial D}=\left.\frac{\partial \varphi_{i}}{\partial \nu}\right|_{\partial D}=0 \\
\int_{D} u_{j} \varphi_{i}=0, \quad \text { for any } i, j=1, \cdots, k .
\end{gathered}
$$

Thus, $\varphi_{i}$ 's are trial functions. Hence, from the Rayleigh-Ritz inequality we have

$$
\Gamma_{k+1} \leq \frac{\int_{D}\left(\Delta \varphi_{i}\right)^{2}}{\int_{D}\left(\varphi_{i}\right)^{2}} .
$$

From (2.3), (2.4) and (2.5), we obtain

$$
\begin{aligned}
\Delta^{2} \varphi_{i} & =\Delta^{2}\left(f u_{i}-\sum_{j=1}^{k} a_{i j} u_{j}\right) \\
= & \Delta\left(\Delta f u_{i}+2 \nabla f \cdot \nabla u_{i}+f \Delta u_{i}\right)-\sum_{j=1}^{k} a_{i j} \Gamma_{j} u_{j} \\
= & (1-n) \Delta u_{i}+2 \Delta\left(\nabla f \cdot \nabla u_{i}\right)+\Delta f \Delta u_{i} \\
& +2 \nabla f \cdot \nabla\left(\Delta u_{i}\right)+f \Delta^{2} u_{i}-\sum_{j=1}^{k} a_{i j} \Gamma_{j} u_{j} \\
& =2(1-n) \Delta u_{i}+2 \Delta\left(\nabla f \cdot \nabla u_{i}\right)+2 \nabla f \cdot \nabla\left(\Delta u_{i}\right)+\Gamma_{i} f u_{i}-\sum_{j=1}^{k} a_{i j} \Gamma_{j} u_{j} .
\end{aligned}
$$

Hence, we infer

$$
\begin{aligned}
& \int_{D}\left(\Delta \varphi_{i}\right)^{2}=\int_{D} \varphi_{i} \Delta^{2} \varphi_{i} \\
& \quad=\Gamma_{i}\left\|\varphi_{i}\right\|^{2}+2 \int_{D} \varphi_{i}\left\{(1-n) \Delta u_{i}+\Delta\left(\nabla f \cdot \nabla u_{i}\right)+\nabla f \cdot \nabla\left(\Delta u_{i}\right)\right\} .
\end{aligned}
$$


Thus,

$$
\begin{aligned}
& \left(\Gamma_{k+1}-\Gamma_{i}\right)\left\|\varphi_{i}\right\|^{2} \\
& \leq 2 \int_{D} \varphi_{i}\left\{(1-n) \Delta u_{i}+\Delta\left(\nabla f \cdot \nabla u_{i}\right)+\nabla f \cdot \nabla\left(\Delta u_{i}\right)\right\} \\
& =2 \int_{D} f u_{i}\left\{(1-n) \Delta u_{i}+\Delta\left(\nabla f \cdot \nabla u_{i}\right)+\nabla f \cdot \nabla\left(\Delta u_{i}\right)\right\} \\
& -2 \sum_{j=1}^{k} a_{i j} \int_{D}\left\{(1-n) \Delta u_{i}+\Delta\left(\nabla f \cdot \nabla u_{i}\right)+\nabla f \cdot \nabla\left(\Delta u_{i}\right)\right\} u_{j} .
\end{aligned}
$$

Defining $b_{i j}$ by

$$
b_{i j}=\int_{D}\left\{(1-n) \Delta u_{i}+\Delta\left(\nabla f \cdot \nabla u_{i}\right)+\nabla f \cdot \nabla\left(\Delta u_{i}\right)\right\} u_{j},
$$

we have

$$
2 b_{i j}=-\left(\Gamma_{i}-\Gamma_{j}\right) a_{i j}=-2 b_{j i} .
$$

In fact,

$$
\begin{aligned}
& b_{i j}=\int_{D}(1-n) \Delta u_{i} u_{j}+\int_{D} \nabla f \cdot \nabla u_{i} \Delta u_{j}-\int_{D}\left(\nabla u_{j} \cdot \nabla f \Delta u_{i}+u_{j} \Delta f \Delta u_{i}\right) \\
& =\int_{D} \nabla f \cdot \nabla u_{i} \Delta u_{j}-\int_{D} \nabla u_{j} \cdot \nabla f \Delta u_{i} .
\end{aligned}
$$

Since

$$
\begin{aligned}
& \int_{D} \nabla f \cdot \nabla u_{i} \Delta u_{j} \\
& =-\int_{D} \Delta f \Delta u_{j} u_{i}-\int_{D} \nabla f \cdot \nabla\left(\Delta u_{j}\right) u_{i} \\
& =(n-1) \int_{D} \Delta u_{j} u_{i}+\int_{D} f \nabla u_{i} \cdot \nabla\left(\Delta u_{j}\right)+\int_{D} f u_{i} \Delta^{2} u_{j} \\
& =(n-1) \int_{D} \Delta u_{j} u_{i}-\int_{D} \nabla f \cdot \nabla u_{i} \Delta u_{j}-\int_{D} f \Delta u_{i} \Delta u_{j}+\Gamma_{j} \int_{D} f u_{i} u_{j},
\end{aligned}
$$

we have

$$
\begin{aligned}
& 2 \int_{D} \nabla f \cdot \nabla u_{i} \Delta u_{j} \\
& =(n-1) \int_{D} \Delta u_{j} u_{i}-\int_{D} f \Delta u_{i} \Delta u_{j}+\Gamma_{j} \int_{D} f u_{i} u_{j} .
\end{aligned}
$$

Furthermore, we know that

$$
\begin{aligned}
& 2 \int_{D} \nabla f \cdot \nabla u_{j} \Delta u_{i} \\
& =(n-1) \int_{D} \Delta u_{i} u_{j}-\int_{D} f \Delta u_{j} \Delta u_{i}+\Gamma_{i} \int_{D} f u_{j} u_{i} .
\end{aligned}
$$

Hence, we infer that

$$
2 b_{i j}=-\left(\Gamma_{i}-\Gamma_{j}\right) a_{i j}
$$


From (2.7) and (2.8), we have

$$
\begin{aligned}
& \left(\Gamma_{k+1}-\Gamma_{i}\right)\left\|\varphi_{i}\right\|^{2} \\
& \leq 2 \int_{D} f u_{i}\left\{(1-n) \Delta u_{i}+\Delta\left(\nabla f \cdot \nabla u_{i}\right)+\nabla f \cdot \nabla\left(\Delta u_{i}\right)\right\}+\sum_{j=1}^{k}\left(\Gamma_{i}-\Gamma_{j}\right) a_{i j}^{2} .
\end{aligned}
$$

Since

we infer that

$$
\int_{D} f u_{i} \Delta u_{i}=(1-n)+2 \int_{D} \nabla f \cdot \nabla u_{i} u_{i}+\int_{D} f u_{i} \Delta u_{i},
$$

$$
\int_{D} u_{i} \nabla f \cdot \nabla u_{i}=\frac{n-1}{2} .
$$

By a direct computation, we have

$$
\begin{aligned}
& \int_{D} f u_{i} \Delta\left(\nabla f \cdot \nabla u_{i}\right) \\
& =\int_{D}\left\{\Delta f u_{i}+2 \nabla f \cdot \nabla u_{i}+f \Delta u_{i}\right\} \nabla f \cdot \nabla u_{i} \\
& =(1-n) \int_{D} u_{i} \nabla f \cdot \nabla u_{i}+2 \int_{D}\left(\nabla f \cdot \nabla u_{i}\right)^{2}+\int_{D} f \Delta u_{i} \nabla f \cdot \nabla u_{i}, \\
& \int_{D} f u_{i} \nabla f \cdot \nabla\left(\Delta u_{i}\right) \\
& =-\int_{D} \Delta u_{i} \nabla f \cdot \nabla\left(f u_{i}\right)-\int_{D} f \Delta f u_{i} \Delta u_{i} \\
& =-\int_{D} u_{i} \Delta u_{i}-\int_{D} f \Delta u_{i} \nabla f \cdot \nabla u_{i}-(1-n) \int_{D} f u_{i} \Delta u_{i} .
\end{aligned}
$$

Therefore, we derive

$$
\begin{aligned}
& 2 \int_{D} f u_{i}\left\{(1-n) \Delta u_{i}+\Delta\left(\nabla f \cdot \nabla u_{i}\right)+\nabla f \cdot \nabla\left(\Delta u_{i}\right)\right\} \\
& =2 \int_{D}\left|\nabla u_{i}\right|^{2}+4 \int_{D}\left(\nabla f \cdot \nabla u_{i}\right)^{2}-(n-1)^{2} \\
& \leq 6 \int_{D}\left|\nabla u_{i}\right|^{2}-(n-1)^{2} .
\end{aligned}
$$

Thus, from $\int_{D}\left|\nabla u_{i}\right|^{2} \leq \Gamma_{i}^{\frac{1}{2}}$, we derive

$$
\begin{aligned}
& \left(\Gamma_{k+1}-\Gamma_{i}\right)\left\|\varphi_{i}\right\|^{2} \\
& \leq 6 \Gamma_{i}^{\frac{1}{2}}-(n-1)^{2}+\sum_{j=1}^{k}\left(\Gamma_{i}-\Gamma_{j}\right) a_{i j}^{2} .
\end{aligned}
$$

Defining

$$
c_{i j}=\int_{D}\left(\nabla f \cdot \nabla u_{i}-\frac{n-1}{2} u_{i}\right) u_{j}
$$

since

$$
\int_{D} \nabla f \cdot \nabla u_{i} u_{j}=(n-1) \int_{D} u_{i} u_{j}-\int_{D} \nabla f \cdot \nabla u_{j} u_{i}
$$


we have

$$
c_{i j}=-\int_{D}\left(\nabla f \cdot \nabla u_{j}-\frac{n-1}{2} u_{j}\right) u_{i}=-c_{j i} .
$$

According to $|\nabla f|^{2}=1$ and

$$
\int_{D} f u_{i} \nabla f \cdot \nabla u_{i}=-\int_{D} \Delta f f u_{i}^{2}-\int_{D} u_{i}^{2}|\nabla f|^{2}-\int_{D} f u_{i} \nabla f \cdot \nabla u_{i},
$$

we have

Hence, we infer that

$$
2 \int_{D} f u_{i} \nabla f \cdot \nabla u_{i}=-1+(n-1) \int_{D} f u_{i}^{2} .
$$

$$
\begin{aligned}
& -2 \int_{D} \varphi_{i}\left(\nabla f \cdot \nabla u_{i}-\frac{n-1}{2} u_{i}\right) \\
& =-2 \int_{D} f u_{i} \nabla f \cdot \nabla u_{i}+2 \sum_{j=1}^{k} a_{i j} \int_{D} u_{j} \nabla f \cdot \nabla u_{i} \\
& =1+2 \sum_{j=1}^{k} a_{i j} \int_{D}\left(\nabla f \cdot \nabla u_{i}-\frac{n-1}{2} u_{i}\right) u_{j} \\
& =1+2 \sum_{j=1}^{k} a_{i j} c_{i j} .
\end{aligned}
$$

On the other hand, for any positive constant $\alpha_{i}$, we have

$$
\begin{aligned}
& 1+2 \sum_{j=1}^{k} a_{i j} c_{i j} \\
& =-2 \int_{D} \varphi_{i}\left(\nabla f \cdot \nabla u_{i}-\frac{n-1}{2} u_{i}\right) \\
& =-2 \int_{D} \varphi_{i}\left(\nabla f \cdot \nabla u_{i}-\frac{n-1}{2} u_{i}-\sum_{j=1}^{k} c_{i j} u_{j}\right) \\
& \leq \alpha_{i}\left\|\varphi_{i}\right\|^{2}+\frac{1}{\alpha_{i}}\left\|\nabla f \cdot \nabla u_{i}-\frac{n-1}{2} u_{i}-\sum_{j=1}^{k} c_{i j} u_{j}\right\|^{2} \\
& =\alpha_{i}\left\|\varphi_{i}\right\|^{2}+\frac{1}{\alpha_{i}}\left\{\left\|\nabla f \cdot \nabla u_{i}-\frac{n-1}{2} u_{i}\right\|^{2}-\sum_{j=1}^{k} c_{i j}^{2}\right\} .
\end{aligned}
$$

If $\Gamma_{k+1}-\Gamma_{i}>0$, we define

$$
\alpha_{i}=\left(\Gamma_{k+1}-\Gamma_{i}\right) \beta_{i} .
$$

Hence, for any $i$ and for $\beta_{i}>0$, we infer that

$$
\begin{aligned}
& \left(\Gamma_{k+1}-\Gamma_{i}\right)^{2}\left(1+2 \sum_{j=1}^{k} a_{i j} c_{i j}\right) \\
& \leq\left(\Gamma_{k+1}-\Gamma_{i}\right)^{3} \beta_{i}\left\|\varphi_{i}\right\|^{2}+\frac{1}{\beta_{i}}\left(\Gamma_{k+1}-\Gamma_{i}\right)\left\{\left\|\nabla f \cdot \nabla u_{i}-\frac{n-1}{2} u_{i}\right\|^{2}-\sum_{j=1}^{k} c_{i j}^{2}\right\} .
\end{aligned}
$$


From (2.10) and

$$
\left\|\nabla f \cdot \nabla u_{i}-\frac{n-1}{2} u_{i}\right\|^{2}=\left\|\nabla f \cdot \nabla u_{i}\right\|^{2}-\frac{(n-1)^{2}}{4} \leq\left\|\nabla u_{i}\right\|^{2}-\frac{(n-1)^{2}}{4},
$$

we have

$$
\begin{aligned}
& \left(\Gamma_{k+1}-\Gamma_{i}\right)^{2}\left(1+2 \sum_{j=1}^{k} a_{i j} c_{i j}\right) \\
& \leq\left(\Gamma_{k+1}-\Gamma_{i}\right)^{2} \beta_{i}\left\{6 \Gamma_{i}^{\frac{1}{2}}-(n-1)^{2}+\sum_{j=1}^{k}\left(\Gamma_{i}-\Gamma_{j}\right) a_{i j}^{2}\right\} \\
& +\frac{1}{\beta_{i}}\left(\Gamma_{k+1}-\Gamma_{i}\right)\left\{\Gamma_{i}^{\frac{1}{2}}-\frac{(n-1)^{2}}{4}\right\}-\frac{1}{\beta_{i}}\left(\Gamma_{k+1}-\Gamma_{i}\right) \sum_{j=1}^{k} c_{i j}^{2} .
\end{aligned}
$$

Since $c_{i j}=-c_{j i}$, we have

$$
\sum_{i, j=1}^{k}\left(\Gamma_{k+1}-\Gamma_{i}\right)^{2} a_{i j} c_{i j}=-\sum_{i, j=1}^{k}\left(\Gamma_{k+1}-\Gamma_{i}\right)\left(\Gamma_{i}-\Gamma_{j}\right) a_{i j} c_{i j} .
$$

Thus,

$$
\begin{array}{r}
2 \sum_{i, j=1}^{k}\left(\Gamma_{k+1}-\Gamma_{i}\right)\left(\Gamma_{i}-\Gamma_{j}\right) a_{i j} c_{i j}-\sum_{i, j=1}^{k}\left(\Gamma_{k+1}-\Gamma_{j}\right)\left(\Gamma_{i}-\Gamma_{j}\right)^{2} \beta_{i} a_{i j}^{2} \\
-\sum_{i, j=1}^{k} \frac{1}{\beta_{i}}\left(\Gamma_{k+1}-\Gamma_{i}\right) c_{i j}^{2} \leq 0 .
\end{array}
$$

According to (2.18), (2.19) and the above inequality, we derive

$$
\begin{aligned}
& \sum_{i, j=1}^{k}\left(\Gamma_{k+1}-\Gamma_{i}\right)^{2} \\
& \leq \sum_{i, j=1}^{k}\left(\Gamma_{k+1}-\Gamma_{i}\right)^{2} \beta_{i}\left\{6 \Gamma_{i}^{\frac{1}{2}}-(n-1)^{2}\right\} \\
& +\sum_{i, j=1}^{k}\left(\Gamma_{k+1}-\Gamma_{i}\right)^{2} \beta_{i}\left(\Gamma_{i}-\Gamma_{j}\right) a_{i j}^{2}+\sum_{i, j=1}^{k}\left(\Gamma_{k+1}-\Gamma_{i}\right) \beta_{i}\left(\Gamma_{i}-\Gamma_{j}\right)^{2} a_{i j}^{2} \\
& +\sum_{i, j=1}^{k} \frac{1}{\beta_{i}}\left(\Gamma_{k+1}-\Gamma_{i}\right)\left\{\Gamma_{i}^{\frac{1}{2}}-\frac{(n-1)^{2}}{4}\right\} .
\end{aligned}
$$

From the variational principle, we can prove that

$$
\Gamma_{i} \geq \lambda_{i}^{2}
$$

where $\lambda_{i}$ denotes the $i^{\text {th }}$ eigenvalue of the Dirichlet eigenvalue problem of the Laplacian on the same domain $D$. Since $\lambda_{1} \geq \frac{(n-1)^{2}}{4}$, putting

$$
\beta_{i}=\frac{1}{\Gamma_{i}^{\frac{1}{2}}-\frac{(n-1)^{2}}{6}} \beta>0,
$$


we have

$$
\begin{aligned}
& \sum_{i, j=1}^{k}\left(\Gamma_{k+1}-\Gamma_{i}\right)^{2} \beta_{i}\left(\Gamma_{i}-\Gamma_{j}\right) a_{i j}^{2}+\sum_{i, j=1}^{k}\left(\Gamma_{k+1}-\Gamma_{i}\right) \beta_{i}\left(\Gamma_{i}-\Gamma_{j}\right)^{2} a_{i j}^{2} \\
& =\sum_{i, j=1}^{k}\left(\Gamma_{k+1}-\Gamma_{i}\right)\left(\Gamma_{k+1}-\Gamma_{j}\right) \beta_{i}\left(\Gamma_{i}-\Gamma_{j}\right) a_{i j}^{2} \\
& =\frac{1}{2} \sum_{i, j=1}^{k}\left(\Gamma_{k+1}-\Gamma_{i}\right)\left(\Gamma_{k+1}-\Gamma_{j}\right)\left(\Gamma_{i}-\Gamma_{j}\right)\left(\beta_{i}-\beta_{j}\right) a_{i j}^{2} \\
& =-\frac{\beta}{2} \sum_{i, j=1}^{k} \frac{\left(\Gamma_{k+1}-\Gamma_{i}\right)\left(\Gamma_{k+1}-\Gamma_{j}\right)\left(\Gamma_{i}^{\frac{1}{2}}+\Gamma_{j}^{\frac{1}{2}}\right)\left(\Gamma_{i}^{\frac{1}{2}}-\Gamma_{j}^{\frac{1}{2}}\right)^{2}}{\left(\Gamma_{i}^{\frac{1}{2}}-\frac{(n-1)^{2}}{6}\right)\left(\Gamma_{j}^{\frac{1}{2}}-\frac{(n-1)^{2}}{6}\right)} a_{i j}^{2} \\
& \leq 0 .
\end{aligned}
$$

From (2.20) and the above inequality, we obtain

$$
\begin{aligned}
& \sum_{i, j=1}^{k}\left(\Gamma_{k+1}-\Gamma_{i}\right)^{2} \leq 6 \beta \sum_{i, j=1}^{k}\left(\Gamma_{k+1}-\Gamma_{i}\right)^{2} \\
& +\frac{1}{\beta} \sum_{i, j=1}^{k}\left(\Gamma_{k+1}-\Gamma_{i}\right)\left\{\Gamma_{i}^{\frac{1}{2}}-\frac{(n-1)^{2}}{4}\right\}\left\{\Gamma_{j}^{\frac{1}{2}}-\frac{(n-1)^{2}}{6}\right\} .
\end{aligned}
$$

Taking

$$
\beta^{2}=\frac{\sum_{i, j=1}^{k}\left(\Gamma_{k+1}-\Gamma_{i}\right)\left\{\Gamma_{i}^{\frac{1}{2}}-\frac{(n-1)^{2}}{4}\right\}\left\{\Gamma_{j}^{\frac{1}{2}}-\frac{(n-1)^{2}}{6}\right\}}{6 \sum_{i, j=1}^{k}\left(\Gamma_{k+1}-\Gamma_{i}\right)^{2}},
$$

we derive

$$
\sum_{i, j=1}^{k}\left(\Gamma_{k+1}-\Gamma_{i}\right)^{2} \leq 24 \sum_{i, j=1}^{k}\left(\Gamma_{k+1}-\Gamma_{i}\right)\left\{\Gamma_{i}^{\frac{1}{2}}-\frac{(n-1)^{2}}{4}\right\}\left\{\Gamma_{j}^{\frac{1}{2}}-\frac{(n-1)^{2}}{6}\right\} .
$$

This finishes the proof of Theorem 1.1.

Proof of Corollary 1.2. Since $\Gamma_{i}^{\frac{1}{2}} \geq \frac{(n-1)^{2}}{4}$, we have

$$
\left\{\Gamma_{i}^{\frac{1}{2}}-\frac{(n-1)^{2}}{4}\right\}\left\{\Gamma_{j}^{\frac{1}{2}}-\frac{(n-1)^{2}}{6}\right\} \leq \Gamma_{i}-\frac{(n-1)^{4}}{16} .
$$

From Theorem 1.1, Corollary 1.2 is proved.

Proof of Theorem 1.4. According to the following recursion formula of Cheng and Yang [7] with $\mu_{i}=\Gamma_{i}-\frac{(n-1)^{4}}{16}$ and $t=\frac{1}{6}$, we have, by making use of the same assertion as in Cheng and Yang [7, that

$$
\mu_{k+1} \leq 25 k^{12} \mu_{1}
$$

that is,

$$
\Gamma_{k+1}-\frac{(n-1)^{4}}{16} \leq 25 k^{12}\left(\Gamma_{1}-\frac{(n-1)^{4}}{16}\right) .
$$


Hence, if $\lim _{D \rightarrow H^{n}(-1)} \Gamma_{1}=\frac{(n-1)^{4}}{16}$, then, for any fixed $k$, we have

$$
\lim _{D \rightarrow H^{n}(-1)} \Gamma_{k}=\frac{(n-1)^{4}}{16} .
$$

This completes the proof of Theorem 1.4.

Recursive Formula (Cheng and Yang [7]). Let $\mu_{1} \leq \mu_{2} \leq \cdots \leq \mu_{k+1}$ be any non-negative real numbers satisfying

$$
\sum_{i=1}^{k}\left(\mu_{k+1}-\mu_{i}\right)^{2} \leq \frac{4}{t} \sum_{i=1}^{k} \mu_{i}\left(\mu_{k+1}-\mu_{i}\right) .
$$

Define

$$
G_{k}=\frac{1}{k} \sum_{i=1}^{k} \mu_{i}, \quad T_{k}=\frac{1}{k} \sum_{i=1}^{k} \mu_{i}^{2}, \quad F_{k}=\left(1+\frac{2}{t}\right) G_{k}^{2}-T_{k} .
$$

Then, we have

$$
F_{k+1} \leq C(t, k)\left(\frac{k+1}{k}\right)^{\frac{4}{t}} F_{k},
$$

where $t$ is any positive real number and

$$
C(t, k)=1-\frac{1}{3 t}\left(\frac{k}{k+1}\right)^{\frac{4}{t}} \frac{\left(1+\frac{2}{t}\right)\left(1+\frac{4}{t}\right)}{(k+1)^{3}}<1 .
$$

\section{REFERENCES}

1. M. S. Ashbaugh, Isoperimetric and universal inequalities for eigenvalues, in Spectral theory and geometry (Edinburgh, 1998), E. B. Davies and Yu Safalov (eds.), London Math. Soc. Lecture Notes, vol. 273, Cambridge Univ. Press, Cambridge, 1999, pp. 95-139. MR1736867 (2001a:35131)

2. I. Chavel, Eigenvalues in Riemannian Geometry, Academic Press, New York, 1984. MR768584 (86g:58140)

3. Z.-C. Chen and C.-L. Qian, Estimates for discrete spectrum of operator with any order, J. China Univ. Sci. Tech. 20 (1990), 259-266. MR.1077287 (92c:35087)

4. Q.-M. Cheng, T. Ichikawa and S. Mametsuka, Estimates for eigenvalues of a clamped plate problem on Riemannian manifolds, J. Math. Soc. Japan 62 (2010), 673-686.

5. Q.-M. Cheng and H. C. Yang, Estimates on eigenvalues of Laplacian, Math. Ann. 331 (2005), 445-460. MR2115463 (2005i:58038)

6. Q.-M. Cheng and H. C. Yang, Inequalities for eigenvalues of a clamped plate problem, Trans. Amer. Math. Soc. 358 (2006), 2625-2635. MR2204047 (2006m:35263)

7. Q.-M. Cheng and H. C. Yang, Bounds on eigenvalues of Dirichlet Laplacian, Math. Ann. 337 (2007), 159-175. MR2262780 (2007k:35064)

8. Q.-M. Cheng and H. C. Yang, Universal estimates for eigenvalues on Riemannian manifolds, J. Differential Equations 247 (2009), 2270-2281. MR.2561278

9. M. Gage, Upper bounds for the first eigenvalue of the Laplace-Beltrami operator, Indiana Univ. Math. J. 29 (1980), 897-912. MR589652 (82b:58095)

10. G. N. Hile and R. Z. Yeh, Inequalities for eigenvalues of the biharmonic operator, Pacific J. Math. 112 (1984), 115-133. MR739143 (85k:35170)

11. S. M. Hook, Domain independent upper bounds for eigenvalues of elliptic operator, Trans. Amer. Math. Soc. 318 (1990), 615-642. MR994167 (90h:35075)

12. M. Levitin and L. Parnovski, Commutators, spectral trance identities, and universal estimates for eigenvalues, J. Functional Analysis 192 (2002), 425-445. MR.1923409 (2003g:47040)

13. H. P. McKean, An upper bound for the spectrum of $\Delta$ on a manifold of negative curvature, J. Differ. Geom. 4 (1970), 359-366. MR0266100(42:1009) 
14. L. E. Payne, G. Polya and H. F. Weinberger, Sur le quotient de deux fréquences propres consécutives, Comptes Rendus Acad. Sci. Paris 241 (1955), 917-919. MR0073046 (17:372d)

15. L. E. Payne, G. Polya and H. F. Weinberger, On the ratio of consecutive eigenvalues, J. Math. and Phys. 35 (1956), 289-298. MR0084696 (18:905c)

16. Q. Wang and C. Xia, Universal bounds for eigenvalues of the biharmonic operator on Riemannian manifolds, J. Functional Analysis 245 (2007), 334-352. MR2311628(2008e:58033)

Department of Mathematics, Faculty of Science and Engineering, Saga University, SAGA 840-8502, JAPAN

E-mail address: cheng@ms.saga-u.ac.jp

Academy of Mathematics and Systematical Sciences, The Chinese Academy of Sciences, Beijing 100080, People's Republic of China

E-mail address: yanghc@math03.math.ac.cn 